\title{
Protection by Recombinant Newcastle Disease Viruses (NDV) Expressing the Glycoprotein (G) of Avian Metapneumovirus (aMPV) Subtype $A$ or $B$ against Challenge with Virulent NDV and aMPV
}

\author{
Qingzhong $\mathrm{Yu}^{1^{*}}$, Jason P. Roth ${ }^{1,2}$, Haixia Hu${ }^{1,3}$, Carlos N. Estevez ${ }^{1,4}$, Wei Zhao ${ }^{1}$, Laszlo Zsak ${ }^{1}$ \\ ${ }^{1}$ Southeast Poultry Research Laboratory, Agricultural Research Services, United States Department of Agriculture, Athens, USA; \\ ${ }^{2}$ Merial Ltd., Athens, USA; ${ }^{3}$ College of Animal Science and Technology, Southwest University, Chongqing, China; ${ }^{4}$ Boehringer-In- \\ gelheim Vetmedica, St. Joseph, USA. \\ Email: *qingzhong.yu@ars.usda.gov
}

Received August $26^{\text {th }}, 2013$; revised September $30^{\text {th }}, 2013$; accepted October $8^{\text {th }}, 2013$

Copyright (C) 2013 Qingzhong Yu et al. This is an open access article distributed under the Creative Commons Attribution License, which permits unrestricted use, distribution, and reproduction in any medium, provided the original work is properly cited.

\begin{abstract}
Avian metapneumovirus (aMPV) and Newcastle disease virus (NDV) are threatening avian pathogens that can cause serious respiratory diseases in poultry worldwide. Vaccination, combined with strict biosecurity practices, has been the recommendation for controlling these diseases in the field. In the present study, we generated NDV LaSota vaccine strain-based recombinant viruses expressing the glycoprotein $(\mathrm{G})$ of aMPV, subtype A or B, using reverse genetics technology. These recombinant viruses, rLS/aMPV-A G and rLS/aMPV-B G, were characterized in cell cultures and evaluated in turkeys as bivalent, next-generation vaccines. The results showed that these recombinant vaccine candidates were slightly attenuated in vivo, yet maintained similar growth dynamics, cytopathic effects, and virus titers in vitro when compared to the parental LaSota virus. The expression of the aMPV G protein in recombinant virus-infected cells was detected by immunofluorescence. Vaccination of turkeys with rLS/aMPV-A G or rLS/aMPV-B G conferred complete protection against velogenic NDV, CA02 strain challenge and partial protection against homologous pathogenic aMPV challenge. These results suggest that the LaSota recombinant virus is a safe and effective vaccine vector and expression of the $\mathrm{G}$ protein alone is not sufficient to provide full protection against aMPV-A or -B infections. Expression of other aMPV-A or -B virus immunogenic protein(s) individually or in conjunction with the $G$ protein may be necessary to induce stronger and more protective immunity against aMPV diseases.
\end{abstract}

Keywords: NDV; aMPV-A and -B; Glycoprotein; Recombinant Virus; Bivalent Vaccine; Turkeys; Protection

\section{Introduction}

Avian metapneumovirus (aMPV) is the causative agent for turkey rhinotracheitis (TRT) and is associated with "swollen head syndrome (SHS)" in chickens, resulting in substantial economic losses to the poultry industry worldwide $[1,2]$. Isolates of aMPV have been classified into four subtypes, A, B, C, and D, based on the level of genetic variations and antigenic differences [2]. The aMPV subtypes $A$ and $B$ are present worldwide, excluding the USA; $\mathrm{C}$ is present mainly in the USA, France, and Korea $[3,4]$; and D has only been reported in France [5].

In European and South American countries, cell cul-

*Corresponding author. ture-attenuated or inactivated vaccines are currently being used to control the diseases caused by the subtypes A and B of aMPV [6-8]. Although these live, attenuated vaccines have been approved and appear to be effective in most countries where the disease is prevalent, several reports have suggested that the stability and safety of some of these live vaccines are of concern [9-12]. Recently in Italy [12] and Brazil [13], field evidence has suggested that the existing vaccines may not fully protect against the circulating field strains of aMPV in these countries. To overcome the problems associated with vaccine safety and stability, efforts have been made to develop inactivated, subunit, virosomal, vectored, or genetically engineered vaccines [14-21]. In contrast to live 
attenuated vaccine, inactivated vaccines are potentially safer, but their protective efficacy remains controversial $[14,17]$. Experimental subunit or vectored vaccines induced varying degrees of protective immunity during clinical trials $[15,16,18,21]$. However, the administration of these non-conventional vaccines may not be practical to large commercial poultry operations.

Newcastle disease virus (NDV) is the etiological agent of Newcastle disease, one of the most serious infectious diseases in poultry. All known strains of NDV are of a single serotype, but have been classified into three different virus pathotypes: velogenic (highly virulent), mesogenic (moderately virulent), and lentogenic (low virulence) [22]. Naturally-occurring lentogenic NDV strains, such as B1, VG/GA, and LaSota strains, are routinely used as live vaccines throughout the world to prevent Newcastle disease [22,23]. These live vaccines induce both strong local and systemic responses and can be readily administered through drinking water supplies or by directly spraying the birds. During the past decade, recombinant NDV viruses have been developed as shuttle-vectors that express foreign antigens, such as avian influenza hemagglutinin (HA) protein, infectious bursal disease virus VP2 protein, and aMPV-C G protein, to protect poultry against NDV and the targeted avian pathogen [24-28].

In this study, we generated LaSota vaccine strainbased recombinant NDV viruses expressing the major surface attachment glycoprotein (G) of aMPV-A or -B using reverse genetics techniques. We evaluated these recombinant viruses in vitro and in vivo for safety, stability, and expression of the $G$ protein for their potential use as bivalent vaccines against NDV and aMPV-A or -B diseases.

\section{Materials and Methods}

\subsection{Cells, Viruses and RNA Preparation}

HEp-2 (CCL-81; ATCC) and DF-1 (CRL-12203; ATCC) cell lines were grown in Dulbecco's Modified Eagle Medium (DMEM, Invitrogen, Carlsbad, CA) supplemented with $10 \%$ fetal bovine serum (FBS, Invitrogen) and antibiotics. The DF- 1 cells were maintained at $37^{\circ} \mathrm{C}$ and $5 \%$ $\mathrm{CO}_{2}$ in DMEM supplemented with $10 \%$ allantoic fluid (AF) from 10-day-old specific-pathogen-free (SPF) chicken embryos for all subsequent infections unless otherwise indicated. The NDV LaSota strain was obtained from ATCC and propagated in 9-day-old SPF chicken embryos. The cell culture-adapted strains of aMPV-A (UK, CVL 14/1) and aMPV-B (Hungary, 657/4) and the velogenic strain of NDV, California 2002 (NDV/CA02; game chicken/US(CA)/S0212676/02) were obtained from the pathogen repository bank at the
Southeast Poultry Research Laboratory (SEPRL, USDAARS, Athens, GA, USA). The pathogenic aMPV-A and -B viruses were obtained from Dr. Kannan Ganapathy (University of Liverpool, UK) and the viruses were prepared from tracheal tissue of virus-infected SPF turkeys as challenge virus stocks and titrated in SPF turkeys for $50 \%$ infective dose $\left(\mathrm{ID}_{50}\right)$ as described previously [29].

Viral RNA was extracted from either AF from NDVinfected chicken embryos or DF-1 cells using the TRIzol-LS reagent according to the manufacturer's instructions (Invitrogen). Total cellular RNA from tracheal tissues was extracted using the MagMAX ${ }^{\mathrm{TM}}$ AI/ND Viral RNA Isolation kit (ABI, Austin, TX) following the manufacturer's procedures.

\subsection{Construction of Recombinant LaSota cDNA Clones Containing the G Gene of aMPV-A or -B}

The infectious LaSota clone (pFLC-LaSota) and subclone (pT-LS MF) were previously generated [30] and used as backbones to construct recombinant cDNA clones containing the G gene of aMPV-A or -B (Figure 1). The open reading frame (ORF) of the $G$ gene of aMPV-A (UK, 14/1) or -B (Hungary, 567/4) was generated by RT-PCR amplification from genomic RNA with paired specific primers using a Superscript ${ }^{\mathrm{TM}}$ III One Step RT-PCR system with Platinum Taq Hi-Fi kit (Invitrogen). Subsequently, the ORF of the aMPV-A or -B G gene was cloned into the intergenic region between the fusion $(\mathrm{F})$ and hemagglutinin-neuraminidase $(\mathrm{HN})$ genes in the pFLC-LaSota vector through a two-step subcloning process using the In-Fusion ${ }^{\circledR}$ PCR cloning kit (Invitrogen). The resulting recombinant clones, designated as pLS/aMPV-A G and pLS/aMPV-B G, respectively, were amplified in Stbl2 cells at $30^{\circ} \mathrm{C}$ for 24 hours and purified using a QIAprep Spin Miniprep kit (Qiagen). The sequences of primers used in the In-Fusion ${ }^{\circledR}$ PCR cloning and $\mathrm{G}$ gene amplification are provided in Table 1.

\subsection{Virus Rescue and Propagation}

Rescue of the recombinant LaSota/aMPV-A or -B G virus was performed by transfection of the full-length cDNA clones and supporting plasmids into HEp-2 cells as described previously [31]. The rescued viruses, which were confirmed by a positive hemagglutination assay (HA) [32], were plaque purified three times in DF-1 cells and finally amplified in SPF chicken embryos three times. The $\mathrm{AF}$ was harvested, aliquoted, and stored at $-80^{\circ} \mathrm{C}$ as a stock. The complete genomic sequences of the rescued viruses were determined by direct sequencing of the RT-PCR products amplified from the viral genomic RNA 


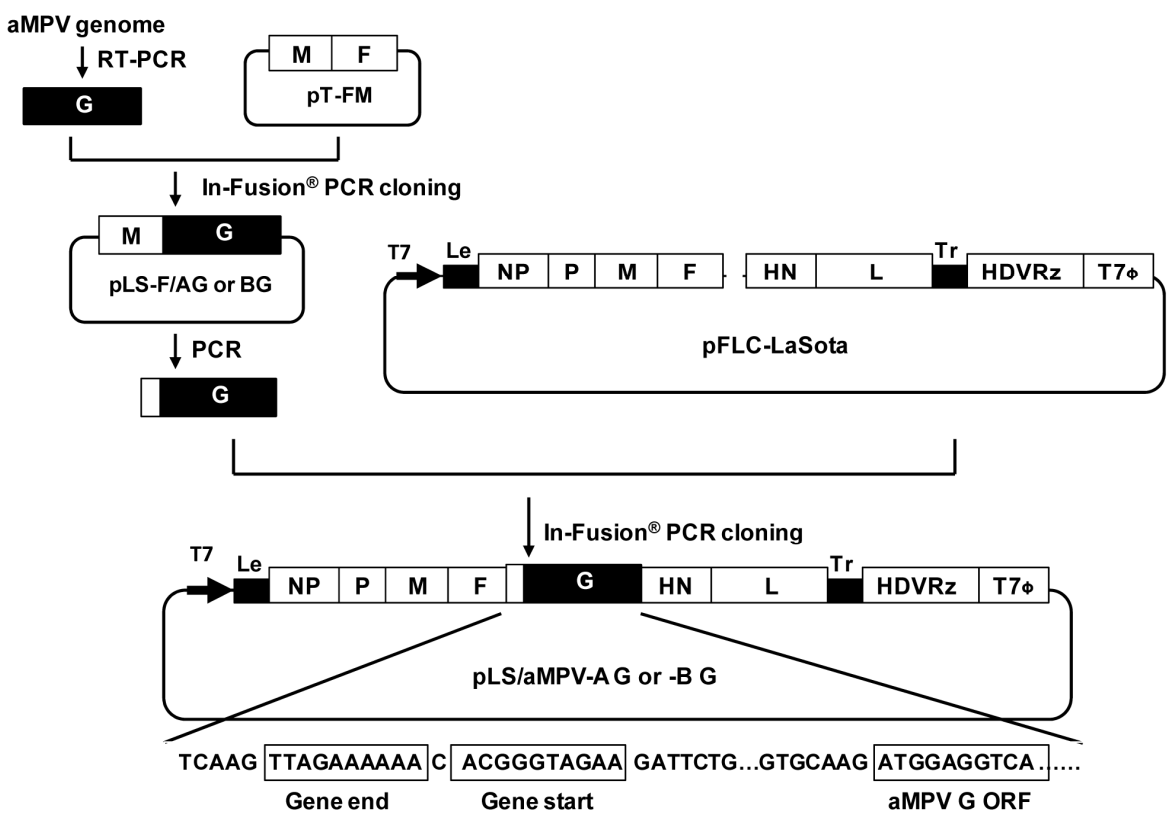

Figure 1. Schematic representation of pLS/aMPV-AG and -BG construction. The open reading frame of the G gene of aMPV-A or -B, amplified from virus genomic RNA, was cloned into the intergenic region between the $F$ and $H N$ genes in the pFLC-LaSota vector through a two-step process using the In-Fusion ${ }^{\circledR}$ PCR cloning kit (Invitrogen). The NDV Gene Start and Gene End signal sequences and the aMPV-A or -B G open reading frame are boxed. The direction of the T7 promoter is indicated by a bold black arrow. HDVRz and T7 $\Phi$ represent the site of the Hepatitis delta virus ribozyme and the T7 terminator sequences, respectively.

Table 1. Primer sequences used in the study.

\begin{tabular}{|c|c|c|}
\hline Primer & Primer Sequence $^{\mathrm{e}}$ & Primer Name \\
\hline $1^{\mathrm{a}}$ & 5'tccaggtgcaagatgGGGTCCAAACTATATATGGCT & aMPV-A NI G F \\
\hline $2^{\mathrm{a}}$ & 5'ctggaattcgccettACTAGTGCAACACCACTCA & aMPV-A NI G R \\
\hline $3^{\mathrm{a}}$ & 5'tccaggtgcaagatgGGGTCAGAGCTCTACATCAT & aMPV-B NI G F \\
\hline $4^{\mathrm{a}}$ & 5'ctggaattcgecettAGCTTATTGACTAGTACAGCACCAC & aMPV-B NI G R \\
\hline $5^{\mathrm{b}}$ & 5'actacaaaaatgtgaGCTGCGTCTCTGAGATTGCG & LS F-M F \\
\hline $6^{\mathrm{b}}$ & 5'gttcctcatctgtgtTCATTAACTAGTGCAACACCACTCA & LS-aMPV-A G RE \\
\hline $7^{\mathrm{b}}$ & 5'gttcctcatctgtgtTTATTGACTAGTACAGCACCA & LS-aMPV-B G RE \\
\hline $8^{\mathrm{c}}$ & 5'CATCTTGCACCTGGAGGGCGCCAAC & pM-F up \\
\hline $9^{c}$ & 5'AAGGGCGAATTCCAGCACACTGGC & pM-F down \\
\hline $10^{\mathrm{c}}$ & 5'TCACATTTTTGTAGTGGCTCTCATC & LS vec F-M up \\
\hline $11^{\mathrm{c}}$ & 5'ACACAGATGAGGAACGAAGGTTTCCCTAATAG & LS vec F down \\
\hline $12^{\mathrm{d}}$ & 5'AGACTCAGTGACTTGGAGTAC & aMPV-A N F19 \\
\hline $13^{\mathrm{d}}$ & 5'TACCGTGATATGGCATCGCT & aMPV-A N R565 \\
\hline $14^{\mathrm{d}}$ & 5'TAAGCTCGCATCCACGGTAGA & aMPV-B N F501 \\
\hline $15^{\mathrm{d}}$ & 5'CTGCATTCCCCAAAACAACACTT & aMPV-B N R979 \\
\hline
\end{tabular}

${ }^{a}$ Primers 1 to 4 were used to RT-PCR amplify the G gene of the aMPV-A or -B strain. ${ }^{b}$ Primers 5 to 7 were used to amplify the cDNA fragments containing the G gene of aMPV-A or -B and the GE and GS sequences of NDV from subclones. ${ }^{\circ}$ Primers 8 to 11 were used to amplify or linearize the pFLC-LaSota or subclone vectors. ${ }^{\mathrm{d}}$ Primers 12 to 15 were used to detect virus replication or viral RNA shedding in tracheal tissues by RT-PCR. ${ }^{\mathrm{e}}$ Nucleotides shown in lower case letters represent homology sequences with a vector backbone, which were used to facilitate the RE independent cloning using the In-Fusion ${ }^{\circledR}$ PCR cloning kit (Clontech). 
as described previously [30].

\subsection{Virus Titration, Pathogenicity, and Growth Dynamics Assays}

Analysis of the recombinant viral stock titers, rLS/ aMPV-A G and rLS/aMPV-B G, were completed using the standard HA test in a 96-well microplate, the 50\% tissue infectious dose $\left(\mathrm{TCID}_{50}\right)$ assay on DF-1 cells, and the $50 \%$ egg infective dose $\left(\mathrm{EID}_{50}\right)$ assay in 9-day-old SPF chicken embryos and compared to the parental LaSota virus [32]. Pathogenicity of the recombinant viruses was assessed by performing the standard mean death time (MDT) and intracerebral pathogenicity index (ICPI) tests and also compared to the parental LaSota virus [32]. Cytopathic effects (CPE) and growth dynamics of the recombinant viruses were examined in DF-1 cells and compared to the parental virus as described previously [30].

\subsection{Immunofluorescence Assay (IFA)}

Expression of the $\mathrm{G}$ protein from DF-1 cells infected with the rLS/aMPV-B G recombinant virus was examined by IFA with anti-aMPV-B chicken serum (kindly provided by Dr. Silke Rautenschlein, University of Vet. Med. Hannover) as described previously [30]. Fluorescence was examined and digitally photographed using an inverted fluorescence microscope at 100X magnifications with matching excitation/emission filters for FITC or Alexa Fluor ${ }^{\circledR} 568$ (Nikon, Eclipse Ti, Melville, NY).

\subsection{Immunization and Challenge Experiments}

Seventy one-day-old SPF turkey poults were randomly divided into seven groups of 10 birds each and housed in Horsfal isolators (Federal Designs, Inc., Comer, GA) with ad libitum access to feed and water in the SEPRL BLS-3E animal facility. Each bird in groups 1, 2 and 3 was inoculated with $100 \mu \mathrm{l}$ PBS via intranasal (IN) and intraocular $(\mathrm{IO})$ routes as controls. Birds in groups 4 and 5 were vaccinated with $100 \mu \mathrm{l}$ of rLS/aMPV-A G $(1.0 \times$ $\left.10^{7} \mathrm{TCID}_{50} / \mathrm{ml}\right)$, and birds in groups 6 and 7 were vaccinated with $100 \mu \mathrm{l}$ of rLS/aMPV-B G $\left(1.0 \times 10^{7} \mathrm{TCID}_{50} /\right.$ $\mathrm{ml})$ per bird via IN/IO routes. At 14 days post-vaccination (DPV), blood samples were collected from each bird to detect serum antibody responses against NDV and aMPV-A or B. Immediately after blood collection, the birds in groups 1,4 , and 6 were challenged with the velogenic NDV/CA02 virus with a dose of $10^{5} \mathrm{EID}_{50} / \mathrm{bird}$ via IN/IO routes as described previously [33]. Mortality of the NDV/CA02-challenged birds was monitored and recorded daily for two weeks. Birds in groups 2, 3, 5, and 7 were challenged with homologous pathogenic aMPV through transmission infection by direct contact with infected birds. Two-week-old SPF turkeys were infected with pathogenic aMPV-A or aMPV-B with a dose of $10^{2}$ $\mathrm{ID}_{50} /$ bird via IN/IO routes. Five of the aMPV-A or $-\mathrm{B}$ virus-infected turkeys were then placed into each corresponding group for the homologous aMPV challenge through direct-contact transmission. The co-mingled birds were monitored daily for clinical signs of aMPV disease for 14 days. Typical clinical signs of the aMPV disease were scored as follows; nasal exudates when squeezed (Score 1), nasal discharge (Score 2), and/or frothy eyes (Score 3), according to the scoring system of Cook et al. [34]. The clinical sign scores post-challenge were statistically analyzed using two-factor ANOVA with a 1\% level of significance between each vaccine treatment and corresponding control group (Microsoft Excel). Tracheal swabs were collected from each aMPV-A or -B viruschallenged birds at 5, 7, and 9 days post-challenge (DPC) for detection of virus shedding.

\subsection{Detection of Immunoresponse and Challenged Virus Shedding}

The NDV-specific serum antibody response was determined using the standard hemagglutination inhibition (HI) test [32] and aMPV subtype-specific serum antibodies were determined by an enzyme-linked immunosorbent assay (ELISA) as described previously, except using sucrose-gradient purified aMPV-A or aMPV-B as an antigen [29,30]. Virus replication or viral RNA shedding from turkey tracheal tissues following challenge with aMPV-A or -B virus was detected by RT-PCR using aMPV-A or -B N gene-specific primers (Table 1) as described previously [29,35].

\section{Results}

\subsection{Generation of the rLS/aMPV-A and -B G Virus}

Two full-length cDNA clones encoding the complete anti-sense genome of the NDV LaSota vaccine strain and the G gene of aMPV-A or -B were constructed through RT-PCR and In-Fusion PCR cloning (Figure 1). The insertion of the transcription "cassettes" containing NDV LaSota intergenic regions and the $G$ gene ORF of aMPV-A or B increased the length of the recombinant clones by 1338 and 1410 nts, respectively. Thus, the total length of pLS/aMPV-A G and pLS/aMPV-B G is 16,524 and 16,596 nts, respectively, and is divisible by 6 abiding by the "Rule of Six" [36]. After co-transfection of the pLS/aMPV-A or -B G clone and supporting plasmids in HEp-2 cells and subsequent amplification in SPF chicken embryonated eggs, the LaSota strain-based recombinant viruses vectoring the $\mathrm{G}$ gene of aMPV-A or -B were rescued, purified and propagated. The fidelity of the rescued viruses was confirmed by sequence analysis 
from RT-PCR products of the viral genome (data not shown).

\subsection{Biological Characterization of the rLS/aMPV-A G and -B G Viruses}

To determine if the additional foreign $\mathrm{G}$ gene affects virus replication of the recombinant rLS/aMPV-A and -B $G$ viruses, pathogenicity and growth dynamics were examined in vitro and in vivo by conducting MDT and ICPI tests and titration assays. As shown in Table 2, the recombinant viruses appeared to be slightly attenuated in day-old chickens with a lower ICPI $(0.0)$ than the parental LaSota strain. The titers of the recombinant viruses grown in either embryonated eggs or in DF-1 cells, as measured by $\mathrm{EID}_{50}, \mathrm{TCID}_{50}$ and $\mathrm{HA}$, were comparable to the titers of the parental LaSota strain (Table 2). They were stable and did not show any apparent changes in MDT and virus titers after 10 passages in SPF chicken embryos (data not shown). In addition, cytopathic effects induced by the rLS/aMPV-A G virus infection were indistinguishable from those seen with the parental LaSota virus in infected DF-1 cells (Figure 2). Finally, no significant differences in the growth kinetics between the
rLS/aMPV-A G, rLS/aMPV-B G and the parental LaSota viruses was detected (Figure 3).

\subsection{Expression of the G Protein by rLS/aMPV -B G}

Expression of the $\mathrm{G}$ protein from aMPV-B $\mathrm{G}$ infected DF-1 cell was examined by IFA using chicken antiaMPV-B serum and FITC-labeled goat anti-chicken IgG. In addition, to pinpoint the location of the expressed $G$ protein in relation to recombinant virus infected DF-1 cells, mouse anti-NDV HN monoclonal antibody (Mab) and Alexa Fluor ${ }^{\circledR} 568$ conjugated goat anti-mouse IgG were also used. As shown in Figure 4, NDV LaSota infected cells were positively stained with mouse antiNDV HN Mab and Alexa-conjugates, but not with chicken anti-aMPV-B serum and FITC conjugate (Figures 4(a) and (b)), demonstrating the specificity of the antibodies and conjugates. When examining rLS/aMPV-B G infected DF-1 cells stained with a mixture of anti-aMPVB/FITC and anti-NDV HN/Alexa 568 antibodies, both green (Figure 4(c)) and red (Figure 4(d)) fluorescence were observed by fluorescence microscopy. After merging both fluorescent images, green and red fluorescence

Table 2. Biological assessments of the NDV/aMPV recombinant viruses.

\begin{tabular}{cccccc}
\hline Virus & MDT $^{\mathrm{a}}$ & $\mathrm{ICPI}^{\mathrm{b}}$ & $\mathrm{HA}^{\mathrm{c}}$ & $\mathrm{EID}_{50}{ }^{\mathrm{d}}$ & $\mathrm{TCID}_{50}{ }^{\mathrm{e}}$ \\
\hline LaSota & $110 \mathrm{hs}$ & 0.15 & 1024 & $6.8 \times 10^{8}$ & $3.5 \times 10^{7}$ \\
rLS/aMPV-A G & $120 \mathrm{hs}$ & 0 & 1024 & $4.2 \times 10^{9}$ & $3.1 \times 10^{8}$ \\
rLS/aMPV-B G & $110 \mathrm{hs}$ & 0 & 1024 & $3.2 \times 10^{9}$ & $9.9 \times 10^{8}$ \\
\hline
\end{tabular}

${ }^{a}$ MDT: Mean death time assay in embryonated chicken eggs. ${ }^{\mathrm{b}} \mathrm{ICPI}$ : Intracerebral pathogenicity index assay in day-old chickens. ${ }^{\mathrm{c}} \mathrm{HA}$ : Hemagglutination assay. ${ }^{\mathrm{d}} \mathrm{EID}_{50}: 50 \%$ egg infective dose assay in embryonated chicken eggs. ${ }^{\mathrm{e}} \mathrm{TCID}_{50}: 50 \%$ tissue infectious dose assay in DF-1 cells.

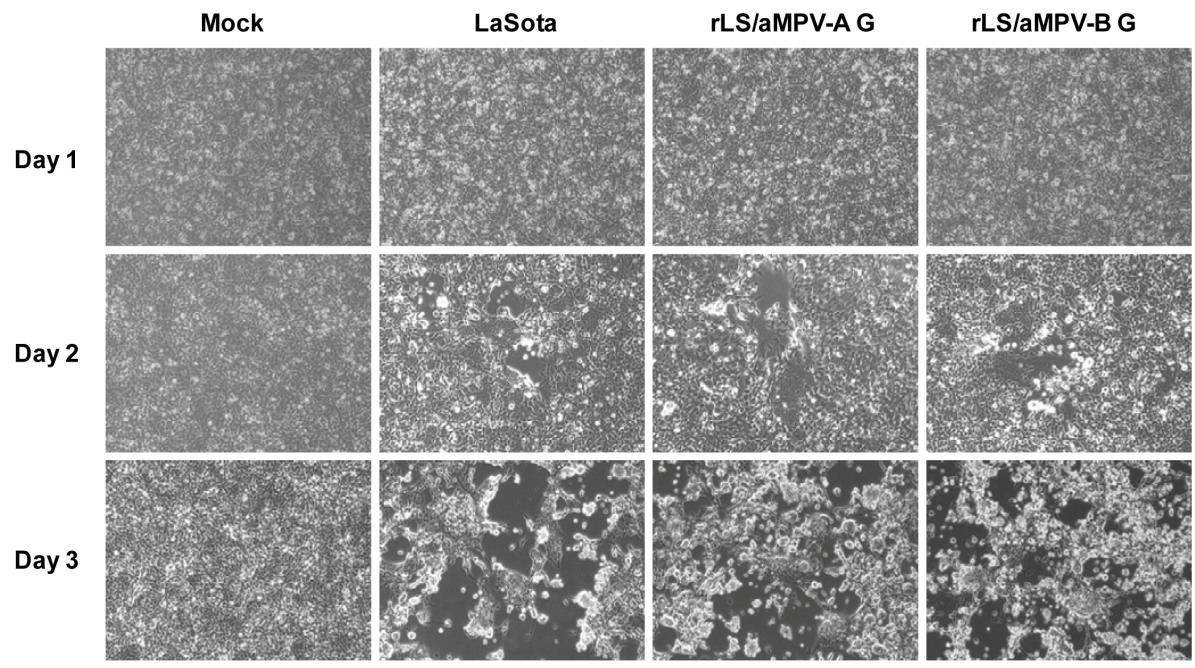

Figure 2. Cytopathic effects induced by the recombinant viruses. Monolayers of DF-1 cells were infected with rLS/aMPV-A G, rLS/aMPV-B G, or LaSota virus at an MOI of 0.001. Mock infection was included as a control. At days 1, 2, and 3 post-infection, infected cells were digitally photographed using an inverted microscope at 100X magnifications (Nikon, Eclipse, $\mathrm{T} i$, Melville, NY). 


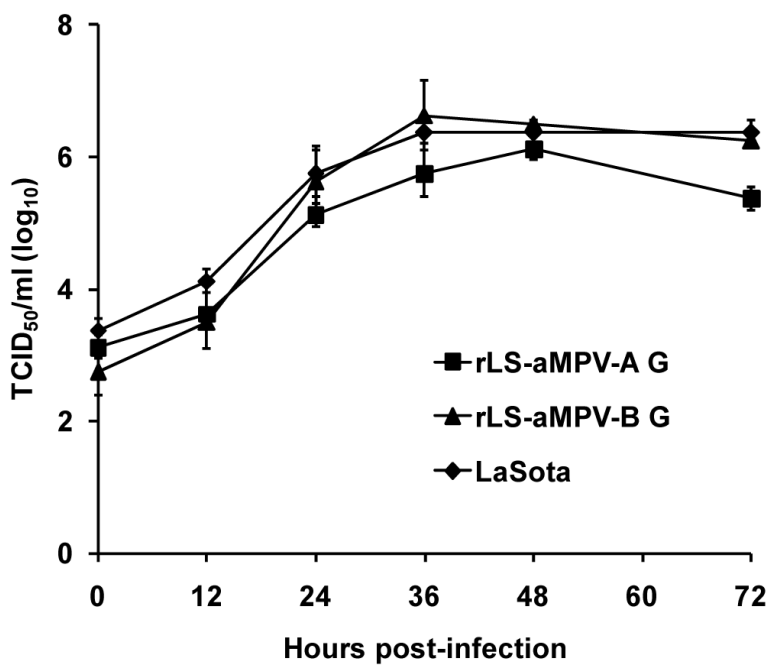

Figure 3. Growth dynamics of the recombinant viruses. DF-1 cells were infected with rLS/aMPV-A G, rLS/aMPV-B G or LaSota strain at an MOI of 0.01 . Every $12 \mathrm{~h}$ post-infection, the cells were harvested. Virus titers at each time point were determined by TCID $_{50}$ titration in DF-1 cells. The mean titer of each time point of duplicate experiments is expressed as $\log _{10}$ $\mathrm{TCID}_{50} / \mathrm{ml}$ with error bars. No significant differences were seen between the viruses.
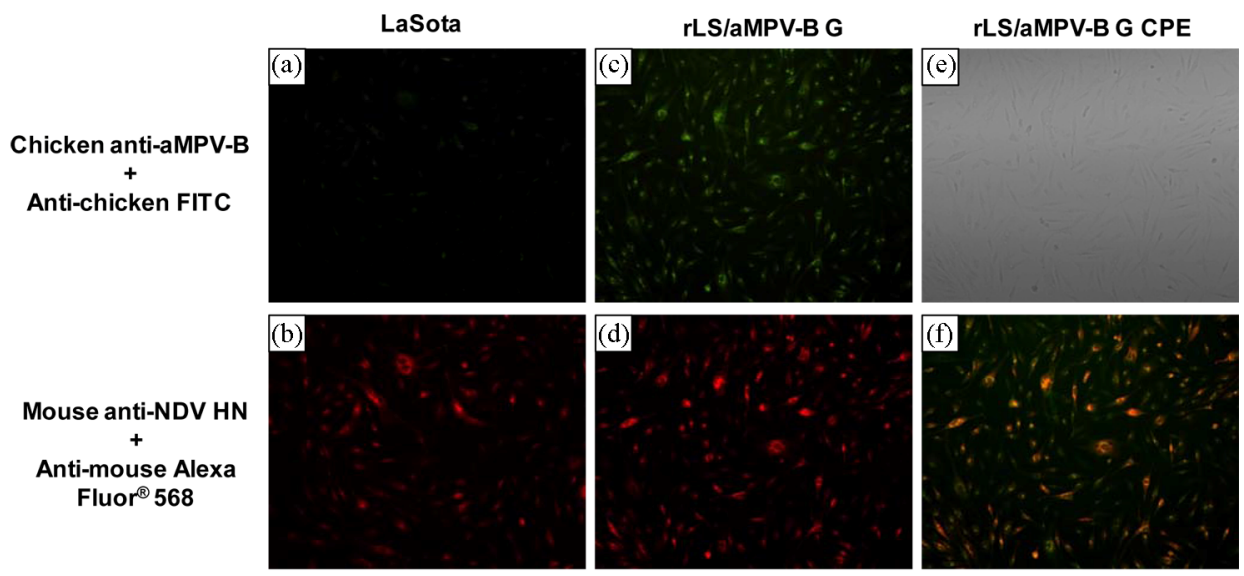

Merged

Figure 4. Detection of aMPV-B G protein expression by IFA. DF-1 cells were infected with LaSota ((a) and (b)) or rLS/aMPV-B G ((c)-(f)) at an MOI of 0.01. At $24 \mathrm{~h}$ post-infection, the infected cells were fixed and stained with a mixture of chicken anti-aMPV-B and mouse anti-NDV Mab followed by a mixture of FITC and Alexa Fluor ${ }^{\circledR} 568$ conjugated antibodies. Fluorescence was examined and digitally photographed using an inverted fluorescence microscope at 100X magnifications under UV light with matching excitation/emission filters for FITC or Alexa Fluor ${ }^{\circledR} 568$ (Nikon, Eclipse, Ti, Melville, NY). Green and red fluorescent images ((c) and (d)) were photographed from the same field of rLS/aMPV-B G-infected cells and merged into one image (f). In addition, viral CPE induced by rLS/aMPV-B G was also photographed from the same field of infected DF-1 cells as the fluorescent images under bright light (e).

co-localized to the same cells (Figure $\mathbf{4}(\mathbf{f})$ ), which corresponded to viral CPE observed in the same field (Figure 4(e)). This result confirms that the aMPV-B G protein is co-expressed with the NDV HN protein from the recombinant virus in the infected cells.

\subsection{Immune Response and Protection against Challenge}

All turkeys that were immunized with either rLS/aMPV-A
G or B G virus produced high NDV-specific HI antibody titers (Table 3) and were completely protected against NDV challenge without showing any clinical sign of disease (Table 3). In contrast, all of the birds in the unvaccinated control group (inoculated with PBS) displayed typical clinical signs of conjunctivitis and severe depression from 2 to 4 DPC and $100 \%$ mortality by 5 DPC. After challenging with the virulent aMPV-A or -B virus through transmission, turkey poults in the control groups (inoculated with PBS) exhibited typical clinical 
Table 3. Serum antibody response of turkeys against NDV following vaccination and survival of turkeys after challenge with a lethal dose of NDV/CA02.

\begin{tabular}{cccc}
\hline \multirow{2}{*}{ Expt. } & Antibody response & \multirow{2}{*}{ Survivors } \\
\cline { 2 - 4 } & Seropositive birds & HI titer $^{\text {a }}$ & $0 / 10$ \\
PBS & $0 / 10$ & 0 & $10 / 10$ \\
rLS/aMPV-A G & $10 / 10$ & $2.9 \pm 1.4$ & $10 / 10$ \\
rLS/aMPV-B G & $10 / 10$ & $4.1 \pm 1.3$ & \\
\hline
\end{tabular}

${ }^{a}$ Hemagglutination inhibition (HI) titer was expressed as $\log _{2}$ of the mean \pm standard deviation.

signs of the disease from 4 DPC, showing nasal exudates when squeezed (Score 1), nasal discharge (Score 2), and/or frothy eyes (Score 3) (Figure 5). The infected birds showed peak clinical signs between 7 - 9 DPC, which gradually decreased in severity thereafter, but at 14 DPC $20 \%$ - $30 \%$ of infected birds still showed some clinical signs. In contrast, turkeys vaccinated with the rLS/aMPV-A $\mathrm{G}$ or $-\mathrm{B} \mathrm{G}$ virus resulted in significantly less severe clinical signs than those in the corresponding control groups (Figure 5, p $<0.01$ ). Most vaccinated birds showed nasal exudates when squeezed or nasal discharge, however these milder clinical signs of the disease disappeared after 11 DPC (Figure 5). Presence of aMPV subtype-specific antibodies in vaccinated turkey sera was not detected by ELISA (data not shown).

Viral RNA shedding, or the presence of the challenge virus (aMPV-A or -B) in the tracheal lumen, was detected in $100 \%$ of the control birds at 5, 7, and $9 \mathrm{DPC}$ (Table 4). Viral shedding of the challenge viruses from corresponding rLS/aMPV-A G or -B G vaccinated birds was somewhat less at 9 DPC when $50 \%$ and $70 \%$ of the birds were negative for viral RNA, respectively (Table 4).

\section{Discussion}

In the present study, we generated and evaluated LaSota strain-based recombinant NDV viruses expressing the $G$ protein of aMPV-A or-B as next-generation, bivalent vaccine candidates. The $\mathrm{G}$ protein of aMPV is thought to be responsible for attachment of the virus particles to the host cell surface receptors to initiate infection. However, the $\mathrm{G}$ deletion or truncation mutants of aMPV were viable in cell cultures, but attenuated in SPF turkeys and induced a weaker immune response than the wild-type virus $[29,37]$, implying that the $G$ protein may play a role in immunogenicity to the natural host. Thus, we selected the $\mathrm{G}$ protein to be expressed by the recombinant NDV vector to investigate the role of the $G$ protein in inducing protective immunity against aMPV challenge, as well as the protective efficacy conferred by the NDV vector against an NDV challenge in turkeys.

Our results showed the safety, stability, and possible application of these recombinant vaccine candidates for use in young turkeys, the most vulnerable population to NDV and aMPV diseases [2,22,38]. Turkeys vaccinated with either rLS/aMPV-A G or -B G virus had comparable levels of NDV-specific $\mathrm{HI}$ antibody response and survived the lethal dose NDV challenge without any clinical sign of disease. To properly evaluate protective efficacy of these vaccine candidates against homologous aMPV challenge, the vaccinated and control birds were challenged with pathogenic aMPV-A or -B through transmission infection to mimic natural infection. It appeared that the infected birds, through transmission, showed clinical signs two days later than birds challenged directly via IN/IO routes, indicating aMPV had a two-day incubation period while spreading through the environment. At 9 DPC with pathogenic aMPV-A or -B, the recombinant virus-vaccinated turkeys showed milder clinical signs and less virus shedding than the birds in the control groups. The lack of detectable aMPV G genespecific antibody response and the partial protection conferred by the recombinant viruses against homologous aMPV challenge suggest that the aMPV G protein is a weak antigen. Our data on the aMPV G protein, inducing partial protective immunity, together with the findings by others on immunogenicity of individual aMPV structural proteins $[18,21]$, demonstrates that a single aMPV protein may not have the capability to induce a strong enough immune response to provide complete protection against aMPV disease. It is reasonable to speculate that co-expression of two or more major structural proteins of the aMPV virus, i.e. the F, G and/or M proteins, perhaps by the NDV vector, may be necessary to induce an enhanced protective immunity against aMPV infection.

In summary, in the present study, we successfully generated NDV/aMPV-A G and -B G recombinant viruses. Turkeys vaccinated with these recombinant viruses were completely protected against velogenic NDV challenge and partially protected against homologous pathogenic aMPV challenge. The results suggest that the aMPV G protein is a weak antigen and other immunegenic components of the virus, most likely the F protein, may be needed and added to the recombinant LaSota vaccine vector in the future to improve the bivalent vac- 


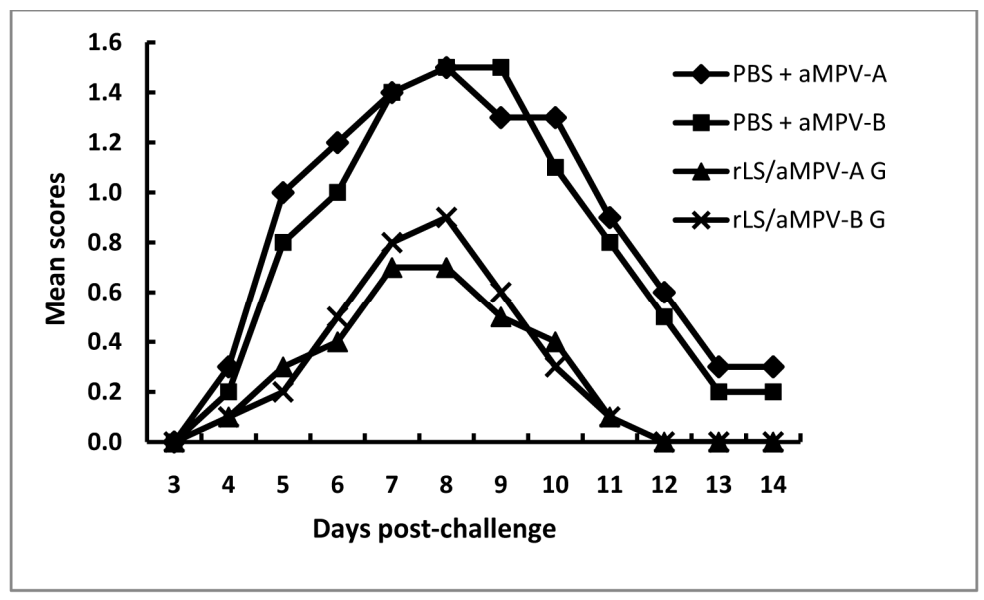

Figure 5. Mean clinical sign scores of vaccinated turkeys after challenge with aMPV-A or -B. Turkey poults were vaccinated with rLS/aMPV-A G, rLS/aMPV-B G, or PBS, and challenged by direct contact transmission with the pathogenic aMPV-A or $-B$ virus, correspondingly, at 14 days post-vaccination. The birds were examined daily for clinical signs and the mean scores from each group of birds were plotted.

Table 4. Viral RNA shedding from trachea following homologous aMPV-A or -B challenge.

\begin{tabular}{cccc}
\hline Expt. & \multicolumn{3}{c}{ Viral RNA shedding ( ${ }^{*}$ of birds $)$} \\
\cline { 2 - 4 } & $5 \mathrm{DPC}^{\mathrm{a}}$ & 7 DPC & 9 DPC \\
\hline PBS & $10 / 10$ & $10 / 10$ & $10 / 10$ \\
rLS/aMPV-A G & $10 / 10$ & $10 / 10$ & $3 / 10$ \\
PBS & $10 / 10$ & $10 / 10$ & $10 / 10$ \\
rLS/aMPV-B G & $10 / 10$ & $10 / 10$ & $5 / 10$ \\
\hline
\end{tabular}

${ }^{\mathrm{a} D P C}$ : days post-challenge.

cine protective efficacy against aMPV infections.

\section{Acknowledgements}

The authors wish to thank Xiuqin Xia and Fenglan Li for excellent technical assistance, Kannan Ganapathy for kindly providing pathogenic aMPV-A and -B viruses, and Silke Rautenschlein and Ron Iorio for a gift of chicken anti-aMPV-B serum and anti-NDV HN monoclonal antibody, respectively. This research was supported by USDA, ARS CRIS project 6612-32000-067-00D. H. Hu and W. Zhao were sponsored by a scholarship from China Scholarship Council.

\section{REFERENCES}

[1] J. K. Cook, "Avian Pneumovirus Infections of Turkeys and Chickens," Veterinary Journal, Vol. 160, No. 2, 2000 , pp. 118-125.

[2] R. E. Gough and R. C. Jones, "Avian Metapneumovirus," In: Y. M. Saif, A. M. Fadly, J. R. Glisson, L. R. McDougald, L. K. Nolan and D. E. Swayne, Eds., Diseases of Poultry, Blackwell Publishing, Ames, 2008, pp. 100-110.

[3] E. Lee, M. S. Song, J. Y. Shin, Y. M. Lee, C. J. Kim, Y. S. Lee, H. Kim and Y. K. Choi, "Genetic Characterization of
Avian Metapneumovirus Subtype C Isolated from Pheasants in a Live Bird Market,"Virus Research, Vol. 128, No. 1-2, 2007, pp. 18-25. http://dx.doi.org/10.1016/j.virusres.2007.03.029

[4] D. Toquin, O. Guionie, V. Jestin, F. Zwingelstein, C. Allee and N. Eterradossi, "European and American Subgroup C Isolates of Avian Metapneumovirus Belong to Different Genetic Lineages," Virus Genes, Vol. 32, No. 1, 2006, pp. 97-103.

http://dx.doi.org/10.1007/s11262-005-5850-3

[5] M. H. Bayon-Auboyer, C. Arnauld, D. Toquin and N. Eterradossi, "Nucleotide Sequences of the F, L and G Protein Genes of Two Non-A/Non-B Avian Pneumoviruses (APV) Reveal a Novel APV Subgroup," Journal of General Virology, Vol. 81, No. 11, 2000, pp. 2723-2733.

[6] J. K. Cook, F. Orthel, S. Orbell, M. A. Woods and M. B. Huggins, “An Experimental Turkey Rhinotracheitis (TRT) Infection in Breeding Turkeys and the Prevention of Its Clinical Effects Using Live-Attenuated and Inactivated TRT Vaccines," Avian Pathology, Vol. 25, No. 2, 1996, pp. 231-243.

http://dx.doi.org/10.1080/03079459608419138

[7] K. Ganapathy, A. Bufton, A. Pearson, S. Lemiere and R. C. Jones, "Vaccination of Commercial Broiler Chicks against Avian Metapneumovirus Infection: A Comparison of Drinking-Water, Spray and Oculo-Oral Delivery Me- 
thods," Vaccine, Vol. 28, No. 23, 2010, pp. 3944-3948. http://dx.doi.org/10.1016/j.vaccine.2010.03.065

[8] R. C. Jones, "Viral Respiratory Diseases (ILT, aMPV Infections, IB): Are They Ever under Control?" British Poultry Science, Vol. 51, No. 1, 2010, pp. 1-11. http://dx.doi.org/10.1080/00071660903541378

[9] P. A. Brown, C. Lupini, E. Catelli, J. Clubbe, E. Ricchizzi and C. J. Naylor, "A Single Polymerase (L) Mutation in Avian Metapneumovirus Increased Virulence and Partially Maintained Virus Viability at an Elevated Temperature," Journal of General Virology, Vol. 92, No. 2, 2011, pp. 346-354. http://dx.doi.org/10.1099/vir.0.026740-0

[10] E. Catelli, M. Cecchinato, C. E. Savage, R. C. Jones and C. J. Naylor, "Demonstration of Loss of Attenuation and Extended Field Persistence of a Live Avian Metapneumovirus Vaccine," Vaccine, Vol. 24, No. 42-43, 2006, pp. 6476-6482.

http://dx.doi.org/10.1016/j.vaccine.2006.06.076

[11] E. Catelli, C. Lupini, M. Cecchinato, E. Ricchizzi, P. A. Brown and C. J. Naylor, "Field Avian Metapneumovirus Evolution Avoiding Vaccine Induced Immunity," Vaccine, Vol. 28, No. 4, 2010, pp. 916-921. http://dx.doi.org/10.1016/j.vaccine.2009.10.149

[12] M. Cecchinato, E. Catelli, C. Lupini, E. Ricchizzi, J. Clubbe, M. Battilani and C. J. Naylor, "Avian Metapneumovirus (AMPV) Attachment Protein Involvement in Probable Virus Evolution Concurrent with Mass Live Vaccine Introduction," Veterinary Microbiology, Vol. 146, No. 1-2, 2010, pp. 24-34. http://dx.doi.org/10.1016/j.vetmic.2010.04.014

[13] J. L. Chacon, M. Mizuma, M. P. Vejarano, D. Toquin, N. Eterradossi, D. P. Patnayak, S. M. Goyal and A. J. Ferreira, "Avian Metapneumovirus Subtypes Circulating in Brazilian Vaccinated and Nonvaccinated Chicken and Turkey Farms," Avian Diseases, Vol. 55, No. 1, 2011, pp. 82-89. http://dx.doi.org/10.1637/9501-081310-Reg.1

[14] R. M. Cha, M. Khatri and J. M. Sharma, "Protection against Avian Metapneumovirus Subtype C in Turkeys Immunized via the Respiratory Tract with Inactivated Virus," Vaccine, Vol. 29, No. 3, 2011, pp. 459-465. http://dx.doi.org/10.1016/j.vaccine.2010.10.074

[15] P. Chary, M. K. Njenga and J. M. Sharma, "Protection by Recombinant Viral Proteins against a Respiratory Challenge with Virulent Avian Metapneumovirus," Veterinary Immunology and Immunopathology, Vol. 108, No. 3-4, 2005, pp. 427-432.

http://dx.doi.org/10.1016/j.vetimm.2005.06.012

[16] D. R. Kapczynski, "Development of a Virosome Vaccine against Avian Metapneumovirus Subtype C for Protection in Turkeys," Avian Diseases, Vol. 48, No. 2, 2004, pp. 332-343. http://dx.doi.org/10.1637/7115

[17] D. R. Kapczynski, L. L. Perkins and H. S. Sellers, "Mucosal Vaccination with Formalin-Inactivated Avian Metapneumovirus Subtype C Does Not Protect Turkeys Following Intranasal Challenge," Avian Diseases, Vol. 52, No. 1, 2008, pp. 28-33. http://dx.doi.org/10.1637/7821-122706-Reg

[18] D. R. Kapczynski and H. S. Sellers, "Immunization of
Turkeys with a DNA Vaccine Expressing Either the F or N Gene of Avian Metapneumovirus," Avian Diseases, Vol. 47, No. 4, 2003, pp. 1376-1383. http://dx.doi.org/10.1637/7033

[19] C. J. Naylor, R. Ling, N. Edworthy, C. E. Savage and A. J. Easton, "Avian Metapneumovirus SH Gene End and G Protein Mutations Influence the Level of Protection of Live-Vaccine Candidates," Journal of General Virology, Vol. 88 , No. 6, 2007, pp. 1767-1775. http://dx.doi.org/10.1099/vir.0.82755-0

[20] C. J. Naylor, C. Lupini and P. A. Brown, "Charged Amino Acids in the AMPV Fusion Protein Have More Influence on Induced Protection than Deletion of the $\mathrm{SH}$ or $\mathrm{G}$ Genes," Vaccine, Vol. 28, No. 41, 2010, pp. 6800-6807. http://dx.doi.org/10.1016/j.vaccine.2010.07.015

[21] Y. Qingzhong, T. Barrett, T. D. Brown, J. K. Cook, P. Green, M. A. Skinner and D. Cavanagh, "Protection against Turkey Rhinotracheitis Pneumovirus (TRTV) Induced by a Fowlpox Virus Recombinant Expressing the TRTV Fusion Glycoprotein (F)," Vaccine, Vol. 12, No. 6, 1994, pp. 569-573.

http://dx.doi.org/10.1016/0264-410X(94)90319-0

[22] D. J. Alexander and D. A. Senne, "Newcastle Disease, Other Avian Paramyxoviruses, and Pneumovirus Infections," In: Y. M. Saif, A. M. Fadly, J. R. Glisson, L. R. McDougald, L. K. Nolan and D. E. Swayne, Eds., Diseases of Poultry, Blackwell Publishing, Ames, 2008, pp. 75-100.

[23] S. B. Hitchner, "History of Biological Control of Poultry Diseases in the USA," Avian Diseases, Vol. 48, No. 1, 2004, pp. 1-8. http://dx.doi.org/10.1637/6100

[24] J. Ge, G. Deng, Z. Wen, G. Tian, Y. Wang, J. Shi, X. Wang, Y. Li, S. Hu, Y. Jiang, C. Yang, K. Yu, Z. Bu and H. Chen, "Newcastle Disease Virus-Based Live Attenuated Vaccine Completely Protects Chickens and Mice from Lethal Challenge of Homologous and Heterologous H5N1 Avian Influenza Viruses," Journal of Virology, Vol. 81 , No. 1, 2007, pp. 150-158. http://dx.doi.org/10.1128/JVI.01514-06

[25] Z. Huang, S. Elankumaran, A. S. Yunus and S. K. Samal, "A Recombinant Newcastle Disease Virus (NDV) Expressing VP2 Protein of Infectious Bursal Disease Virus (IBDV) Protects against NDV and IBDV," Journal of Virology, Vol. 78, No. 18, 2004, pp. 10054-10063. http://dx.doi.org/10.1128/JVI.78.18.10054-10063.2004

[26] B. Nayak, S. N. Rout, S. Kumar, M. S. Khalil, M. M. Fouda, L. E. Ahmed, K. C. Earhart, D. R. Perez, P. L. Collins and S. K. Samal, "Immunization of Chickens with Newcastle Disease Virus Expressing H5 Hemagglutinin Protects against Highly Pathogenic H5N1 Avian Influenza Viruses," PLoS One, Vol. 4, No. 8, 2009, p. E6509. http://dx.doi.org/10.1371/journal.pone.0006509

[27] M. S. Park, J. Steel, A. Garcia-Sastre, D. Swayne and P. Palese, "Engineered Viral Vaccine Constructs with Dual Specificity: Avian Influenza and Newcastle Disease," Proceedings of the National Academy of Sciences of the United States of America, Vol. 103, No. 21, 2006, pp. 8203-8208. http://dx.doi.org/10.1073/pnas.0602566103

[28] J. Veits, D. Wiesner, W. Fuchs, B. Hoffmann, H. Gran- 
zow, E. Starick, E. Mundt, H. Schirrmeier, T. Mebatsion, T. C. Mettenleiter and A. Romer-Oberdorfer, "Newcastle Disease Virus Expressing H5 Hemagglutinin Gene Protects Chickens against Newcastle Disease and Avian Influenza," Proceedings of the National Academy of Sciences of the United States of America, Vol. 103, No. 21, 2006, pp. 8197-8202.

http://dx.doi.org/10.1073/pnas.0602461103

[29] Q. Yu, C. N. Estevez, M. Song, D. R. Kapczynski and L. Zsak, "Generation and Biological Assessment of Recombinant Avian Metapneumovirus Subgroup C (aMPV-C) Viruses Containing Different Length of the G Gene," Virus Research, Vol. 147, No. 2, 2010, pp. 182-188. http://dx.doi.org/10.1016/j.virusres.2009.10.021

[30] H. Hu, J. P. Roth, C. N. Estevez, L. Zsak, B. Liu and Q. $\mathrm{Yu}$, "Generation and Evaluation of a Recombinant Newcastle Disease Virus Expressing the Glycoprotein (G) of Avian Metapneumovirus Subgroup C as a Bivalent Vaccine in Turkeys," Vaccine, Vol. 29, No. 47, 2011, pp. 8624-8633.

http://dx.doi.org/10.1016/j.vaccine.2011.09.007

[31] C. N. Estevez, D. J. King, B. Seal and Q. Yu, "Evaluation of Newcastle Disease Virus Chimeras Expressing the Hemagglutinin-Neuraminidase Protein of Velogenic Strains in the Context of a Mesogenic Recombinant Virus Backbone," Virus Research, Vol. 129, No. 2, 2007, pp. 182190. http://dx.doi.org/10.1016/j.virusres.2007.07.008

[32] D. J. Alexander, "Newcastle Disease Virus and Other Avian Paramyxoviruses," In: D. Swayne, J. R. Glisson, M. W. Jackwood, J. E. Pearson and W. M. Reed, Eds., A Laboratory Manual for the Isolation and Identification of Avian Pathogens, American Association of Avian Pathologists, Kennett Square, 1998, pp. 156-163.
[33] D. R. Kapczynski and D. J. King, "Protection of Chickens against Overt Clinical Disease and Determination of Viral Shedding Following Vaccination with Commercially Available Newcastle Disease Virus Vaccines upon Challenge with Highly Virulent Virus from the California 2002 Exotic Newcastle Disease Outbreak," Vaccine, Vol. 23, No. 26, 2005, pp. 3424-3433. http://dx.doi.org/10.1016/j.vaccine.2005.01.140

[34] J. K. Cook, M. M. Ellis, C. A. Dolby, H. C. Holmes, P. M. Finney and M. B. Huggins, "A Live Attenuated Turkey Rhinotracheitis Virus Vaccine: 1. Stability of the Attenuated Strain," Avian Pathology, Vol. 18, No. 3, 1989, pp. 511-522. http://dx.doi.org/10.1080/03079458908418623

[35] Q. Yu, C. N. Estevez, J. P. Roth, H. Hu and L. Zsak, "Deletion of the M2-2 Gene from Avian Metapneumovirus Subgroup C Impairs Virus Replication and Immunogenicity in Turkeys," Virus Genes, Vol. 42, No. 3, 2011, $\mathrm{pp}$. 339-346.

http://dx.doi.org/10.1007/s11262-011-0577-9

[36] D. Kolakofsky, L. Roux, D. Garcin and R. W. Ruigrok, "Paramyxovirus mRNA Editing, the 'Rule of Six' and Error Catastrophe: A Hypothesis," Journal of General Virology, Vol. 86, No. 7, 2005, pp. 1869-1877. http://dx.doi.org/10.1099/vir.0.80986-0

[37] D. Govindarajan, S. H. Kim and S. K. Samal, "Contribution of the Attachment G Glycoprotein to Pathogenicity and Immunogenicity of Avian Metapneumovirus Subgroup C," Avian Diseases, Vol. 54, No. 1, 2010, pp. 5966. http://dx.doi.org/10.1637/8991-071409-Reg.1

[38] R. C. Jones, C. Baxter-Jones, G. P. Wilding and D. F. Kelly, "Demonstration of a Candidate Virus for Turkey Rhinotracheitis in Experimentally Inoculated Turkeys," Veterinary Record, Vol. 119, No. 24, 1986, pp. 599-600. 